Pacific Journal of Mathematics

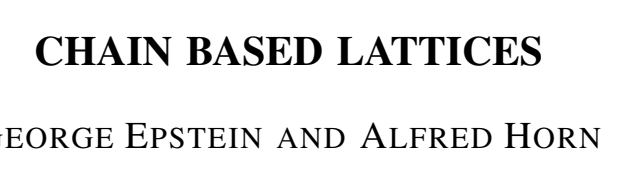




\section{CHAIN BASED LATTICES}

\section{G. Epstein ANd A. HorN}

In recent years several weakenings of Post algebras have been studied. Among these have been $P_{0}$-lattices by T. Traczyk, Stone lattice of order $n$ by T. Katrinak and A. Mitschke, and $P$-algebras by the present authors. Each of these system is an abstraction from certain aspects of Post algebras, and no two of them are comparable. In the present paper, the theory of $P_{0}$-lattices will be developed further and two new systems, called $P_{1}$-lattices and $P_{2}$-lattices are introduced. These systems are referred to as chain based lattices. $P_{2}$-lattices form the intersection of all three weakenings mentioned above. While $P$-algebras and weaker systems such as $L$-algebras, Heyting algebras, and $B$-algebras, do not require any distinguished chain of elements other than 0,1 , chain based lattices require such a chain.

Definitions are given in $\S 1$. A $P_{0}$-lattice is a bounded distributive lattice $A$ which is generated by its center and a finite subchain containing 0 and 1 . Such a subchain is called a chain base for $A$. The order of a $P_{0}$-lattice $A$ is the smallest number of elements in a chain base of $A$. In $\S 2$, properties of $P_{0}$-lattices are given which are used in later sections. If a $P_{0}$-lattice $A$ is a Heyting algebra, then it is shown in $\S 3$, that there exists a unique chain base $0=e_{0}<e_{1}<\cdots<$ $e_{n-1}=1$ such that $e_{i+1} \rightarrow e_{i}=e_{i}$ for all $i>0$. A $P_{0}$-lattice with such a chain base is called a $P_{1}$-lattice. Every $P_{1}$-lattice of order $n$ is a Stone lattice of order $n$. If a $P_{1}$-lattice is pseudo-supplemented then it is called a $P_{2}$-lattice. It turns out that $P_{2}$-lattices of order $n$ are direct products of finitely many Post algebras whose maximum order is $n$. In $\S 4$, properties of $P_{2}$-lattices are studied. In $\S 5$, equational axioms are given for $P_{2}$-lattices. $P_{2}$-lattices share many of the properties of Post algebras and have application to computer science. Among examples of $P_{2}$-lattices are direct products of finitely many $p$-rings. These further remarks on $P_{2}$-lattices are in $\S 6$. In $\S 7$, prime ideals in $P_{0}$-lattices are studied. It is shown that the order of a $P_{0}$-lattice is one more than the number of elements in a chain of prime ideals of maximum length. A characterization of $P_{1}$-lattices by properties of their prime ideals is given. Such a characterization of $P_{2}$-lattices is also indicated.

1. Definitions. We use $\phi$ for the empty set. Let $A$ be a distributive lattice which is bounded, that is, has a largest element 1 and a smallest element 0 . The dual of $A$ is denoted by $A^{d}$. The 
complement of $x$ is denoted by $\bar{x}$ or $-x$. The center of $A$ is the set $B$ of all complemented elements of $A$. We use $x \vee y$ for the join, and $x \wedge y$ or $x y$ for the meet of two elements $x, y$ in $A . \quad x \rightarrow y$ denotes the largest $z \in A$ (if it exists) such that $x z \leqq y . \quad A$ is called a Heyting algebra if $x \rightarrow y$ exists for all $x, y \in A . \quad-x=x \rightarrow 0$ is called the pseudo-complement of $x$ (when it exists). An element $x$ is called dense if $\neg x=0 . \quad A$ is called pseudo-complemented if $\neg x$ exists for all $x \in A . \quad x \Rightarrow y$ denotes the largest $z \in B$ such that $x z \leqq y . \quad A$ is called a $B$-algebra if $x \Rightarrow y$ exists for all $x, y \in A . \quad ! x=1 \Rightarrow x$ is called the pseudo-supplement of $x . A$ is called pseudo-supplemented if $! x$ exists for all $x \in A$.

A Stone lattice is a pseudo-complemented lattice satisfying the identity $-x \vee \neg \neg x=1$. An L-algebra is a Heyting algebra satisfying $(x \rightarrow y) \vee(y \rightarrow x)=1$. A P-algebra is a $B$-algebra satisfying $(x \Rightarrow y) \vee(y \Rightarrow x)=1$. We denote the interval $\{z: x \leqq z \leqq y\}$ by $[x, y]$. $A$ is an $L$-algebra if and only if every interval in $A$ is a Stone lattice $[1,3.11]$. The identity $x \rightarrow(y \vee z)=(x \rightarrow y) \vee(x \rightarrow z)$ is satisfied in an $L$-algebra. The identity $x \Rightarrow(y \vee z)=(x \Rightarrow y) \vee(x \Rightarrow z)$ is satisfied in a $P$-algebra.

2. $P_{0}$-lattices. Let $A$ be a bounded distributive lattice and let $B$ be a Boolean subalgebra of the center of $A$. A chain base of $A$ is a finite sequence $0=e_{0} \leqq e_{1} \leqq \cdots \leqq e_{n-1}=1$ such that $A$ is generated by $B \cup\left\{e_{0}, \cdots, e_{n-1}\right\}$. If $A$ has a chain base then $A$ is called a $P_{0}$-lattice [13], in which case every element $x \in A$ can be written in the form

$$
x=\bigvee_{i=1}^{n-1} b_{i} e_{i},
$$

where $b_{i} \in B$. If $b_{i} \geqq b_{i+1}$ for all $i$, then (1) is called a monotone representation (abbreviated mon. rep.) of $x$. If $b_{i} b_{j}=0$ for $i \neq j$, then (1) is called a disjoint representation (disj. rep.) of $x$. Every element in a $P_{0}$-lattice has both a mon. rep. and a disj. rep.

LEMMA 2.1. If (1) is a mon. rep. of $x$ and $y=\mathrm{V}_{i} c_{i} e_{i}$ is a mon. rep., then $x \vee y=\mathrm{V}_{i}\left(b_{i} \vee c_{i}\right) e_{i}$ and $x y=\mathrm{V}_{i} b_{i} c_{i} e_{i}$ are mon. reps.

Proof. This follows from the distributivity of $A$.

The following theorem shows that $B$ must coincide with the center of the $P_{0}$-lattice $A$, and gives a method for constructing $P_{0}$-lattices.

TheOREM 2.2. Let $A$ be a bounded distributive lattice. Let 
$B$ be a subalgebra of the center of $A$ and let $0=e_{0} \leqq \cdots \leqq e_{n-1}=1$. If $A_{0}$ is the sublattice generated by $B \cup\left\{e_{0}, \cdots, e_{n-1}\right\}$, and $B_{0}$ is the center of $A_{0}$, then $B_{0}=B$.

Proof. Let $x=\mathrm{V}_{j} b_{j} e_{j}$ be a disj. rep. of an element $x \in B_{0}$. For each $i, x b_{i}=b_{i} e_{i}$ is in $B_{0}$. Let $\mathrm{V}_{j} c_{j} e_{j}=0$ be a mon. rep. of the complement of $b_{i} e_{i}$. Then $b_{i} e_{i} \bigvee_{j} c_{j} e_{j}=0$ implies $b_{i} c_{i} e_{i}=0$, hence $b_{i} e_{i} \leqq b_{i} \bar{c}_{i}$. Also $1=b_{i} e_{i} \vee \bigvee_{j} c_{j} e_{j}$ implies $1 \leqq e_{i} \vee c_{i}$, hence $b_{i} \bar{c}_{i} \leqq b_{i} e_{i}$. Thus $b_{i} e_{i}=b_{i} \bar{c}_{i} \in B$ for all $i$, and so $x \in B$.

Definition 2.3. A $P_{0}$-lattice $A$ is said to be of order $n$ if $n$ is the smallest integer such that $A$ has a chain base with $n$ terms.

Lemma 2.4. If $\left\langle A ; e_{0}, \cdots, e_{n-1}\right\rangle$ is a $P_{0}$-lattice, then $\left\langle A^{d} ; e_{n-1}\right.$, $\left.\cdots, e_{0}\right\rangle$ is a $P_{0}$-lattice. $A^{d}$ has the same order as $A$.

Proof. This is obvious by inspection.

THEOREM 2.5. If $\left\langle A ; e_{0}, \cdots, e_{n-1}\right\rangle$ is a $P_{0}$-lattice with center $B$ and $A^{\prime}=\left[e_{i}, e_{j}\right]$, where $i \leqq j$, then $\left\langle A^{\prime} ; e_{i}, \cdots, e_{j}\right\rangle$ is a $P_{0}$-lattice with center $B^{\prime}=\left\{e_{i} \vee b e_{j}: b \in B\right\}$. If $e_{i}=f_{0} \leqq \cdots \leqq f_{r-1}=e_{j}$ is a chain base of $A^{\prime}$, then $e_{0}, \cdots, e_{i-1}, f_{0}, \cdots, f_{r-1}, e_{j+1}, \cdots, e_{n-1}$ is a chain base of $A$. If $A$ has order $n$, then $A^{\prime}$ has order $j-i+1$.

Proof. Let $x=\bigvee_{k=1}^{n-1} b_{k} e_{k}$ be a mon. rep. of an element $x \in A^{\prime}$. Then

$$
x=\left(e_{i} \vee x\right) e_{j}=e_{i} \vee \bigvee_{k=i+1}^{j} b_{k} e_{k}=\bigvee_{k=i+1}^{j}\left(e_{i} \vee b_{k} e_{j}\right) e_{k} .
$$

$B^{\prime}$ is clearly a subalgebra of the center of $A^{\prime}$. Therefore by $2.2, B^{\prime}$ is the center of the $P_{0}$-lattice $\left\langle A^{\prime} ; e_{i}, \cdots, e_{j}\right\rangle$. The remaining parts of the theorem hold because if $i \leqq k \leqq j$, then $e_{k}$ is in the sublattice generated by $B^{\prime} \cup\left\{f_{0}, \cdots, f_{r-1}\right\}$.

Lemma 2.6. Let $A$ be a bounded distributive lattice with center $B$, and $x, y, z \in A$.

(i) If $x \rightarrow z$ and $y \rightarrow z$ exist, then $(x \vee y) \rightarrow z=(x \rightarrow z)(y \rightarrow z)$.

(ii) If $z \rightarrow x$ and $z \rightarrow y$ exist, then $z \rightarrow x y=(z \rightarrow x)(z \rightarrow y)$.

(iii) If $x \rightarrow y$ exists, $b \in B$ and $c \in B$, then $b x \rightarrow(c \vee y)=$ $\bar{b} \vee c \vee(x \rightarrow y)$.

(iv) If $x \Rightarrow z$ and $y \Rightarrow z$ exist, then $(x \vee y) \Rightarrow z=(x \Rightarrow z)(y \Rightarrow z)$.

(v) If $z \Rightarrow x$ and $z \Rightarrow y$ exist, then $z \Rightarrow x y=(z \Rightarrow x)(z \Rightarrow y)$.

(vi) If $x \Rightarrow y$ exists, $b \in B$ and $c \in B$, then $b x \Rightarrow(c \vee y)=$ $\bar{b} \vee c \vee(x \Rightarrow y)$. 
Proof. The proof is straightforward.

LEMmA 2.7. If $a_{2} \leqq \cdots \leqq a_{m}$ and $b_{1} \geqq \cdots \geqq b_{m-1}$ are elements of a distributive lattice, then $\bigvee_{j=1}^{m-1} a_{j+1} b_{j}=a_{m} b_{1} \bigwedge_{j=2}^{m-1}\left(a_{j} \vee b_{j}\right)$.

Proof. This is easily proved by induction.

Theorem 2.8. Let $\left\langle A ; e_{0}, \cdots, e_{n-1}\right)$ be a $P_{0}$-lattice with center $B$. Then the following are equivalent:

(i) $e_{i} \Rightarrow 0$ exists for all $i$.

(ii) $-e_{i}$ exists for all $i$.

(iii) $A$ is pseudo-complemented.

(iv) $A$ is a Stone lattice.

(v) Each $x \in A$ has a mon. rep. $x=\mathrm{V}_{i} b_{i} e_{i}$ such that $b_{1} \leqq c_{1}$ for every mon. rep. $x=\mathrm{V}_{i} c_{i} e_{i}$.

Proof. (i) implies (ii): Let $x e_{i}=0$ and suppose $x=\mathrm{V}_{j} b_{j} e_{j}$ is a mon. rep. of $x$. Then $b_{j} e_{j}=0$ for $j \leqq i$, while if $j>i$, then $b_{j} e_{i}=0$, so $b_{j} \leqq e_{i} \Rightarrow 0$. Hence $x \leqq e_{i} \Rightarrow 0$. Therefore, $\neg e_{i}$ exists and equals $e_{i} \Rightarrow 0$.

(ii) implies (iii): If $x=\mathrm{V}_{i} b_{\imath} e_{i}$ is a mon. rep., then by 2.6(i) and 2.6(iii), $\neg x$ exists and equals $\Lambda_{i}\left(\bar{b}_{i} \vee \neg e_{i}\right)$. If follows from 2.7 that

$$
\neg x=\bigvee_{i=1}^{n-1} \bar{b}_{i} \neg e_{i-1}
$$

(iii) implies (iv): If $x, y \in A$, then by 2.1 and (2), $\neg(x y)=$ $\neg x \vee-y$. This implies that $A$ is a Stone lattice [8].

(iv) implies (v): If $x=\mathrm{V}_{i} c_{i} e_{i}$ is any mon. rep., then $\overline{c_{1}} x=0$, so $\bar{c}_{1} \leqq \neg x$, hence $x \leqq \neg \neg x \leqq c_{1}$. Therefore $x=\mathrm{V}_{i}\left(c_{i} \neg \neg x\right) e_{i}$. If we set $b_{i}=c_{i} \neg \neg x$, we get a mon. rep. in which $b_{1}=\neg \neg x$.

(v) implies (i): Let $e_{i}=\mathrm{V}_{j} b_{j} e_{j}$ be a mon. rep. of $e_{i}$ having the property stated in $(\mathrm{v})$. Then $\bar{b}_{1} e_{\imath}=0$. If $b \in B$ and $b e_{i}=0$, then $e_{i} \leqq \bar{b}$, so $e_{i}=\mathrm{V}_{j} \bar{b} b_{j} e_{j}$. By hypothesis, $\bar{b} b_{1} \geqq b_{1}$. Therefore $b \leqq \bar{b}_{1}$, and so $e_{i} \Rightarrow 0=\bar{b}_{1}$.

Lemma 2.9. If $A$ is a bounded distributive lattice, then $A^{d}$ is a Stone lattice if and only if $A$ is pseudo-supplemented and $!(x \vee y)=$ $! x \vee ! y$ for all $x, y \in A$.

Proof. It is easily verified that the pseudo-complement of $x$ in $A^{d}$ is $\overline{! x}$ in this case.

Theorem 2.10. Let $\left\langle A ; e_{0}, \cdots, e_{n-1}\right\rangle$ be a $P_{0}$-lattice. Then the 
following are equivalent:

(i) !ei exists for all $i$.

(ii) $A$ is pseudo-supplemented and $!(x \vee y)=! x \vee ! y$ for all $x, y \in A$.

(iii) Each $x \in A$ has a mon. rep. $\bigvee_{i} b_{i} e_{i}$ such that $b_{n-1} \geqq c_{n-1}$ for every mon. rep. $x=\mathrm{V}_{i} c_{i} e_{i}$.

Proof. This is derived from 2.8 by using 2.4 and 2.9 .

THEOREM 2.11. Let $\left\langle A ; e_{0}, \cdots, e_{n-1}\right\rangle$ be a pseudo-complemented $P_{0}$-lattice. Then $A$ has a chain base $0=f_{0} \leqq f_{1} \leqq \cdots \leqq f_{n-1}=1$ such that $f_{1}$ is the smallest dense element of $A$. If $0=g_{0} \leqq \cdots \leqq$ $g_{r-1}=1$ is any chain base of $A$ such that $g_{1}$ is dense, then $g_{1}=f_{1}$ and for any mon. rep. $x=\bigvee_{j=1}^{r-1} b_{j} g_{j}$, we have $\neg x=\bar{b}_{1}$.

Proof. Let $f_{0}=0, f_{1}=\bigvee_{i=1}^{n-1}\left(\neg e_{i-1}\right) e_{i}$, and $f_{i}=e_{i} \vee f_{1}$ for $i \geqq 2$. By (2), $\neg f_{1}=\mathrm{V}_{i} \neg \neg e_{i-1} \neg e_{i}=0$. Also $f_{i}=e_{i} \vee \mathrm{V}_{j>i} e_{j} \neg e_{j-1}$. Therefore $f_{i} \neg \neg e_{i}=e_{i}$, since $\neg \neg e_{i} \neg e_{j-1} \leqq \neg \neg e_{i} \neg e_{i}=0$ for $j>i$. If $x=\mathbf{V}_{i} b_{i} e_{i}$ is any element of $A$, then $x=\mathbf{V}_{i}\left(b_{i} \neg \neg e_{i}\right) f_{i}$. Thus $f_{0}, \cdots, f_{n-1}$ is a chain base of $A$. Let $g_{0}, \cdots, g_{r-1}$ be a chain base of $A$ such that $g_{1}$ is dense. If $x=\mathrm{V}_{j} b_{j} g_{j}$ is a mon. rep., then $\neg x=\bar{b}_{1}$ by (2). So if $x$ is dense, then $x \geqq g_{1}$. Thus $g_{1}=f_{1}$ is the smallest dense element of $A$.

\section{3. $P_{1}$-lattices.}

Theorem 3.1. Let $\left\langle A ; e_{0}, \cdots, e_{n-1}\right\rangle$ be a $P_{0}$-lattice with center $B$. Then the following are equivalent:

(i) $e_{i} \rightarrow e_{j}$ exists for all $i, j$.

(ii) $A$ is a Heyting algebra.

(iii) $A$ is an L algebra.

Proof. (i) implies (ii): If $x=\mathrm{V}_{i} b_{i} e_{i}$ and $y=\mathrm{V}_{i} c_{i} e_{i}$ are mon. reps., then by 2.7, $y=\bigwedge_{i=1}^{n-1}\left(c_{i} \vee e_{i-1}\right)$. Therefore by 2.6, $x \rightarrow y$ exists and equals $\Lambda_{i, j}\left(\bar{b}_{i} \vee c_{j} \vee\left(e_{i} \rightarrow e_{j}\right)\right)$.

(ii) implies (iii): Let $x=\mathrm{V}_{i} b_{i} e_{i}, y=\mathrm{V}_{i} c_{2} e_{i}$ be mon. reps. Then $x \rightarrow y=\Lambda_{i}\left(b_{i} e_{i} \rightarrow y\right) \geqq \Lambda_{i}\left(\bar{b}_{i} \vee c_{i}\right)$. Therefore, $\quad(x \rightarrow y) \vee(y \rightarrow x) \geqq$ $\Lambda_{i}\left(\bar{b}_{i} \vee c_{i}\right) \vee \Lambda_{i}\left(b_{i} \vee \bar{c}_{i}\right)=\Lambda_{i, j}\left(\bar{b}_{i} \vee b_{j} \vee c_{i} \vee \bar{c}_{j}\right)=1$ since $\bar{b}_{i} \vee b_{j}=1$ for $i \geqq j$, and $c_{i} \vee \bar{c}_{j}=1$ for $i<j$.

(iii) implies (i): This is obvious.

Definition 3.2. A $P_{1}$-lattice $\left(A ; e_{0}, \cdots, e_{n-1}\right\rangle$ is a $P_{0}$-lattice to- 
gether with a chain base such that $e_{i+1} \rightarrow e_{i}=e_{i}$. It follows that $e_{i} \rightarrow e_{j}=e_{j}$ for $i>j$ and $e_{i} \rightarrow e_{j}=1$ for $i \leqq j$, so that (i) of 3.1 holds.

THEOREM 3.3. If $\left\langle A ; e_{0}, \cdots, e_{n-1}\right\rangle$ is a $P_{0}$-lattice and $A$ is a Heyting algebra, then there exists a chain base $0=f_{0} \leqq \cdots \leqq f_{n-1}=1$ such that $\left\langle A ; f_{0}, \cdots, f_{n-1}\right\rangle$ is a $P_{1}$-lattice.

Proof. This is obvious for $n=1,2$. Suppose $n>2$ and the statement holds for $n-1$. By 2.11, we may assume $e_{1}$ is dense. Let $A^{\prime}=\left[e_{1}, 1\right]$. By $2.5,\left\langle A^{\prime} ; e_{1}, \cdots, e_{n-1}\right\rangle$ is a $P_{0}$-lattice. If $x, y \in A^{\prime}$, then $x \rightarrow y \in A^{\prime}$. Therefore by the induction hypothesis, there exists a sequence $e_{1}=f_{1} \leqq \cdots \leqq f_{n-1}=1$ such that $\left\langle A^{\prime} ; f_{1}, \cdots, f_{n-1}\right\rangle$ is a $P_{1}$-lattice. If we set $f_{0}=0$, then by $2.5,\left\langle A ; f_{0}, \cdots, f_{n-1}\right\rangle$ is a $P_{1}$ lattice.

Theorem 3.4. Let $\left\langle A ; e_{0}, \cdots, e_{n-1}\right\rangle$ be a $P_{1}$-lattice. Then for some $m \geqq 1,0=e_{0}<e_{1}<\cdots<e_{m-1}=e_{m}=\cdots=1$. A has order m. For each $i, e_{i+1}$ is the smallest dense element of $\left[e_{i}, 1\right]$. Thus $e_{0}, \cdots, e_{m-1}$ is the unique strictly increasing chain such that $\left\langle A ; e_{0}, \cdots, e_{m-1}\right\rangle$ is a $P_{1}$-lattice. If $x=\bigvee_{i=1}^{n-1} b_{i} e_{i}$ is a mon. rep., then $e_{i} \vee b_{i+1}=\left(x \rightarrow e_{i}\right) \rightarrow e_{i}, 0 \leqq i<n-1$. If $x=\bigvee_{i=1}^{n-1} b_{i} e_{i}$ is a disj. rep., and $y=\bigvee_{i=1}^{n-1} c_{i} e_{i}$ is a mon. rep., then $x \rightarrow y=y \vee \bigvee_{i=0}^{n-1} b_{i} c_{i}$, where $b_{0}=\bigwedge_{i=1}^{n-1} \bar{b}_{i}, c_{0}=1$.

Proof. If $m$ is the first integer such that $e_{m}=e_{m-1}$, then $e_{m-1}=$ $e_{m} \rightarrow e_{m-1}=1$. Since $e_{i+1}$ is dense in $\left[e_{i}, 1\right]$ it follows from 2.5 and 2.11 that $e_{i+1}$ is the smallest dense element of $\left[e_{i}, 1\right]$. Using 3.3 , it follows that $A$ has order $m$.

If $x=\bigvee_{i=1}^{n-1} b_{i} e_{i}$ is a mon. rep., then $x \vee e_{i}=\bigvee_{k=i+1}^{n-1}\left(e_{i} \vee b_{k}\right) e_{k}$. Applying 2.11 to $\left[e_{i}, 1\right]$, we find $\left(x \vee e_{i}\right) \rightarrow e_{i}=e_{i} \vee \bar{b}_{i+1}$. Since $\left(x \vee e_{i}\right) \rightarrow$ $e_{i}=x \rightarrow e_{i}$, it follows by 2.6 that $\left(x \rightarrow e_{i}\right) \rightarrow e_{i}=e_{i} \vee b_{i+1}$.

To prove the last statement, we observe that

$$
\begin{aligned}
e_{i} \rightarrow y & =\bigvee_{j=1}^{n-1}\left(e_{i} \rightarrow c_{j} e_{j}\right)=\bigvee_{j=0}^{n-1}\left(e_{i} \rightarrow c_{j}\right)\left(e_{i} \rightarrow e_{j}\right) \\
& =\bigvee_{j=1}^{i-1} c_{j} e_{j} \vee \bigvee_{j=i}^{n-1} c_{j}=y \vee c_{i} \text { for } 1 \leqq i \leqq n-1
\end{aligned}
$$

Therefore,

$$
\begin{aligned}
x \rightarrow y & =\bigwedge_{i=1}^{n-1}\left(b_{i} e_{i} \rightarrow y\right)=\bigwedge_{i=1}^{n-1}\left(\bar{b}_{i} \vee c_{i} \vee y\right)=y \vee \bigwedge_{i-1}^{n-1}\left(\bar{b}_{i} \vee c_{i}\right) \\
& =y \vee \bigvee_{i=0}^{n-1} b_{i} c_{i},
\end{aligned}
$$


where the last equality is easily proved by induction.

DEFINITION 3.5. A Stone lattice $\left\langle A ; e_{0}, \cdots, e_{n-1}\right\rangle$ of order $n$ is an $L$-algebra $A$ in which there exists a chain $0=e_{0}<e_{1}<\cdots<$ $e_{n-1}=1$ such that $e_{i+1}$ is the smallest dense element of $\left[e_{i}, 1\right]$. If $B_{i}$ is the center of $\left[e_{i}, 1\right]$, let $h_{i}: B_{i} \rightarrow B_{i+1}$ be the Boolean homomorphism defined by $h_{i}(x)=x \vee e_{i+1}$, with $B_{0}=B$. These definitions are in [11].

TheOREM 3.6. $\left\langle A ; e_{0}, \cdots, e_{n-1}\right\rangle$ is a $P_{1}$-lattice of order $n$, if and only if $\left\langle A ; e_{0}, \cdots, e_{n-1}\right\rangle$ is a Stone lattice of order $n$ such that $h_{i}$ is onto $B_{i+1}$ for each $i \geqq 0$.

Proof. If $\left\langle A ; e_{0}, \cdots, e_{n-1}\right\rangle$ is a $P_{1}$-lattice of order $n$, then it is a Stone lattice of order $n$ by 3.4, and $h_{i}$ is onto by 2.5. Conversely, suppose $\left\langle A ; e_{0}, \cdots, e_{n-1}\right\rangle$ is a Stone lattice of order $n$ and $h_{i}$ is onto $B_{i+1}$ for each $i$. Then $B_{i}=\left\{b \vee e_{i}: b \in B\right\}$ by 2.5. It was proved in $[11,3.4]$, that if $x \in A$, then $x=\bigwedge_{i=0}^{n-2} x_{i}$, where $x_{i} \in B_{i}$. Therefore $\left\langle A ; e_{0}, \cdots, e_{n-1}\right\rangle$ is a $P_{1}$-lattice.

THEOREM 3.7. If $A$ is a Heyting algebra with center $B, 0=e_{0} \leqq$ $\cdots \leqq e_{n-1}=1, e_{i+1}$ is the smallest dense element of $\left[e_{i}, 1\right]$, and if whenever $i<j$, the center of $\left[e_{i}, e_{j}\right]$ is $\left\{e_{i} \vee b e_{j}: b \in B\right\}$, then $\left\langle A ; e_{0}, \cdots\right.$, $e_{n-1}>$ is a $P_{1}$-lattice.

Proof. The point of this theorem is that the condition that $A$ is an $L$-algebra is replaced by the condition that $A$ is a Heyting algebra such that the center of $\left[e_{i}, e_{j}\right]$ is $\left\{e_{i} \vee b e_{j}: b \in B\right\}$, for all $i<j$. We omit details of proof since this theorem is not used in what follows.

\section{4. $P_{2}$-lattices.}

Definition 4.1. $x=\bigvee_{i=1}^{n-1} b_{i} e_{i}$ is called the highest monotone representation (hi. mono. rep.) of $x$ if for every mon. rep. $\mathbf{V}_{i=1}^{n-1} c_{i} e_{i}$ of $x$, the relation $b_{\imath} \geqq c_{i}$ holds for all $i$. The lowest monotonic representation (lo. mon. rep.) is defined in a similar manner.

Theorem 4.2. Let $\left\langle A ; e_{0}, \cdots, e_{n-1}\right\rangle$ be a $P_{0}$-lattice. Then the following are equivalent:

(i) $e_{i} \Rightarrow e_{j}$ exists for all $i, j$.

(ii) $e_{i} \rightarrow e_{j}$ and $! e_{i}$ exist for all $i, j$.

(iii) every $x \in A$ has a hi. mon. rep.

(iv) every $x \in A$ has a lo. mon. rep. 
(v) $A$ is a B-algebra.

(vi) $A$ is a P-algebra.

The hi. mon. rep. of $x$ is $\mathbf{V}_{i}\left(e_{i} \Rightarrow x\right) e_{i}$, and the lo. mon. rep. of $x$ is $\mathbf{V}_{i}\left(\overline{x \Rightarrow e_{i-1}}\right) e_{i}$.

Proof. The equivalence of (i), (v), and (vi) is proved exactly as in the proof of 3.1. By [7], $A$ is a $P$-algebra if and only if $A$ is a pseudo-supplemented $L$-algebra in which $!(x \vee y)=! x \vee ! y$ for all $x, y$. Therefore, by 3.1 and 2.10 , (ii) is equivalent to (vi).

To prove (iii) implies (i), let $V_{j} b_{j} e_{j}$ be the hi. mon. rep. of $e_{i}$. Then $b_{i+1} e_{i+1} \leqq e_{i}$. Let $b \in B, b e_{i+1} \leqq e_{i}$. Thus $e_{1} \vee \cdots \vee e_{i} \vee b e_{i+1}$ is a mon. rep. of $e_{i}$. Therefore $b_{i+1} \geqq b$, which proves $b_{i+1}=e_{i+1} \Rightarrow e_{i}$. Hence if $i>j, e_{i} \Rightarrow e_{j}=\bigwedge_{k=i}^{j-1}\left(e_{k} \Rightarrow e_{k+1}\right)$, and for $i \leqq j, e_{i} \Rightarrow e_{j}=1$.

To prove (vi) implies (iii), let $x=\mathbf{V}_{i} b_{i} e_{i}$ be any mon. rep. Then $e_{i} \Rightarrow x \geqq e_{i} \Rightarrow b_{i} e_{i} \geqq b_{i}$. Also $e_{i}\left(e_{i} \Rightarrow x\right) \leqq x$. Therefore,

$$
x \geqq \mathrm{~V}_{i} e_{i}\left(e_{i} \Rightarrow x\right) \geqq \mathrm{V}_{i} b_{i} e_{i}=x
$$

Thus $\mathrm{V}_{i} e_{i}\left(e_{i} \Rightarrow x\right)$ is the hi. mon. rep. of $x$.

The equivalence of (iv) and (vi) is a consequence of the equivalence of (iii) and (vi), since the dual of a $P$-algebra is a $P$-algebra. The formula for the lo. mon. rep. is obtained by duality, for if $x=\mathrm{V}_{i} b_{i} e_{i}$ is a mon. rep., then $x=\Lambda_{i}\left(b_{i} \vee e_{i-1}\right)$.

Definition 4.3. A $P_{2}$-lattice is a $P_{1}$-lattice $\left\langle A ; e_{0}, \cdots, e_{n-1}\right\rangle$ such that $! e_{i}$ exists for all $i$.

Using 2.2, it is easy to construct a $P_{1}$-lattice which is not a $P_{2}$-lattice.

THeOREM 4.4. If $\left\langle A ; e_{0}, \cdots, e_{n-1}\right\rangle$ is a $P_{0}$-lattice of order $n$ and $A$ is a B-algebra, then there exists a unique chain $f_{0}, \cdots, f_{n-1}$ such that $\left\langle A ; f_{0}, \cdots, f_{n-1}\right\rangle$ is a $P_{2}$-lattice.

Proof. This follows from 3.3, 3.4, and 4.2.

THEOREM 4.5. Let $\left\langle A ; e_{0}, \cdots, e_{n-1}\right\rangle$ be a $P_{2}$-lattice. Then

(i) Every $x \in A$ has a unique mon. rep. $\mathrm{V}_{i} D_{i}(x) e_{i}$ such that $D_{n-1}(x)=! x$. This representation is also the hi. mon. rep. of $x$.

(ii) Every $x \in A$ has a unique disj. rep. $\mathrm{V}_{i} C_{i}(x) e_{i}$ such that $C_{n-1}(x)=! x$.

(iii) $D_{i}(x)=e_{i} \Rightarrow x, C_{i}(x)=D_{i}(x)-D_{i+1}(x)$ and for $i<n-1$, $C_{i}(x)=\left(x \Rightarrow e_{i}\right)\left(e_{i} \Rightarrow x\right)-!\left(x e_{i}\right)$.

(iv) $D_{i}(x \vee y)=D_{i}(x) \vee D_{i}(y), D_{i}(x y)=D_{i}(x) D_{i}(y)$

(v) $x \Rightarrow y=\bigvee_{i=0}^{n-1} C_{i}(x) D_{i}(y)$, where $D_{0}(y)=1$ and $C_{0}(x)=1$ $D_{1}(x)$. 
Proof. (i) Let $x=\mathrm{V}_{j} b_{j} e_{j}$ be a mon. rep. such that $b_{n-1}=! x$. If $i>j$, then $e_{i} \Rightarrow e_{j}=!\left(e_{i} \rightarrow e_{j}\right)=! e_{j}$. Therefore,

$$
\begin{aligned}
e_{i} \Rightarrow x & =\bigvee_{j}\left(e_{i} \Rightarrow b_{j} e_{j}\right)=\bigvee_{j}\left(e_{i} \Rightarrow b_{j}\right)\left(e_{i} \Rightarrow e_{j}\right) \\
& =\bigvee_{j<i} b_{j} ! e_{j} \bigvee \bigvee_{j \geqq i} b_{j}=b_{i},
\end{aligned}
$$

since $\bigvee_{j=1}^{n-1} b_{j} ! e_{j}=! x \leqq b_{i}$. We set $D_{i}(x)=e_{i} \Rightarrow x$ for $0 \leqq i \leqq n-1$. By 4.2, the hi. mon. rep. of $x$ is $\mathrm{V}_{i} D_{i}(x) e_{i}$, and $D_{n-1}(x)=1 \Rightarrow x=! x$.

(ii) Follows from (i), with $C_{i}(x)=D_{\imath}(x)-D_{i+1}(x)$.

(iii) For $0 \leqq i<n-1$,

$$
\begin{aligned}
x \Rightarrow e_{i} & =\bigwedge_{j}\left(D_{j}(x) e_{j} \Rightarrow e_{i}\right)=\bigwedge_{j}\left(\overline{D_{j}(x)} \vee\left(e_{j} \Rightarrow e_{i}\right)\right) \\
& \left.=\bigwedge_{j>i} \overline{\left(D_{j}(x)\right.} \vee ! e_{i}\right)=\overline{D_{i+1}(x)} \vee ! e_{i} .
\end{aligned}
$$

Therefore $\left(x \Rightarrow e_{i}\right)\left(e_{i} \Rightarrow x\right)=C_{i}(x) \vee D_{i}(x) ! e_{i}$. Since $D_{i}(x) ! e_{i} \leqq e_{i}\left(e_{i} \Rightarrow\right.$ $x) \leqq x$, we have $D_{i}(x) ! e_{i} \leqq ! x=D_{n-1}(x)$. Hence $D_{i}(x) ! e_{i}=! x ! e_{i}=!\left(x e_{i}\right)$. Also $C_{i}(x) ! x=C_{i}(x) C_{n-1}(x)=0$. Therefore,

$$
C_{i}(x)=\left(x \Rightarrow e_{i}\right)\left(e_{i} \Rightarrow x\right)-!\left(x e_{i}\right) .
$$

(iv) follows immediately from $D_{i}(x)=e_{i} \Rightarrow x$.

(v) By 3.4, $x \rightarrow y=y \vee \bigvee_{i=0}^{n-1} C_{i}(x) D_{i}(y)$. Therefore,

$$
x \Rightarrow y=! y \vee \bigvee_{i=0}^{n-1} C_{i}(x) D_{i}(y)=\bigvee_{i=0}^{n-1} C_{i}(x) D_{i}(y),
$$

since $\bigvee_{i=0}^{n-1} C_{i}(x) D_{i}(y) \geqq D_{n-1}(y) \bigvee_{i=0}^{n-1} C_{i}(x)=! y$.

THEOREM 4.6. The following are equivalent:

(i) $\left\langle A ; e_{0}, \cdots, e_{n-1}\right\rangle$ is a $P_{2}$-lattice of order $n$.

(ii) $\left\langle A ; e_{0}, \cdots, e_{n-1}\right\rangle$ is a Stone lattice of order $n$, the homomorphisms $h_{i}$ of 3.5 are onto, and the kernel of $h_{i}$ is a principal ideal for each $i$.

(iii) $\left\langle A ; e_{0}, \cdots, e_{n-1}\right\rangle$ is a Stone lattice of order $n$ and $A^{d}$ is a Stone lattice.

Proof. The equivalence of (i) and (ii) follows from 3.6 and 2.9, using the fact that the kernel of $h_{i}$ is a principal ideal if and only if $! e_{i+1}$ exists. The equivalence of (ii) and (iii) was proved in [11].

The following is the dual of the definition given in [5].

Definition 4.7. A Post algebra is a $P_{2}$-algebra $\left\langle A ; e_{0}, \cdots, e_{n-1}\right\rangle$ such that $! e_{n-2}=0$; that is, $e_{n-2}$ is dense in $A^{d}$. Note that $A$ has order $n$, unless $A=\{0\}$. 
Theorem 4.8. If $\left\langle A ; e_{0}, \cdots, e_{n-1}\right\rangle$ is a $P_{0}$-lattice then the following are equivalent:

(i) $A$ is a Post algebra.

(ii) every element $x \in A$ has a unique mon. rep.

(iii) $e_{i} \Rightarrow e_{i-1}=0$ for all $i>0$.

Proof. This was proved in [13].

Lemma 4.9. If $\left\langle A_{j}\right.$; $\left.e_{j 0}, \cdots, e_{j\left(n_{j}-1\right)}\right\rangle$ is a $P_{r}$-lattice for $j \in J$, where $r=0,1$, or $2, A=\prod_{j \in J} A_{j}, n=\max \left\{n_{j}: j \in J\right\}<\infty$, and $e_{j k}$ is defined to be $e_{j\left(n_{j}-1\right)}$ for $k>n_{j}$, then $\left\langle A ; e_{0}, \cdots, e_{n-1}\right\rangle$ is a $P_{r}$-lattice, where $e_{i}=\left\langle e_{j i}: j \in J\right\rangle$.

Proof. This is obvious.

Lemma 4.10. If $\left\langle A ; e_{0}, \cdots, e_{n-1}\right)$ is a $P_{0}$-lattice, $B$ is a distributive lattice and $f: A \rightarrow B$ is a lattice homomorphism onto, then $\left\langle B ; f\left(e_{0}\right), \cdots, f\left(e_{n-1}\right)\right\rangle$ is a $P_{0}$-lattice. If $\left\langle A ; e_{0}, \cdots, e_{n-1}\right\rangle$ is a $P_{1}$-lattice and $f: A \rightarrow B$ is a Heyting homomorphism onto, then $\left\langle B ; f\left(e_{0}\right), \cdots\right.$, $f\left(e_{n-1}\right)>$ is a $P_{1}$-lattice.

Proof. This is easy to verify.

TheOREM 4.11. Let $A$ be a finite distributive lattice then the following are equivalent:

(i) $A$ is a $P_{0}$-lattice.

(ii) $A$ is a P-algebra.

(iii) $A$ is a direct product of chains.

(iv) $A$ has a chain base $e_{0}, \cdots, e_{n-1}$ such that $\left\langle A ; e_{0}, \cdots, e_{n-1}\right\rangle$ is a $P_{2}$-lattice.

Proof. (i) implies (ii): Since $A$ is finite, $A$ is a pseudo-supplemented Heyting algebra. By $4.2, A$ is a $P$-algebra.

(ii) implies (iii) was proved in [7].

(iii) implies (iv): If $A$ is a finite chain $0=a_{0}<\cdots<a_{n-1}=1$, then $\left\langle A ; a_{0}, \cdots, a_{n-1}\right\rangle$ is a $P_{2}$-lattice. Therefore (iv) follows by 4.9 . (iv) implies (i) is obvious.

A finite chain with $n$ elements has exactly one chain base with $n$ terms. If $\left\langle A ; e_{0}, \cdots, e_{n-1}\right\rangle$ and $\left\langle B ; f_{0}, \cdots, f_{m-1}\right\rangle$ are $P_{0}$-lattices of orders $n$ and $m$ respectively, where $n<m$, then $A \times B$ has more than one chain base. In addition to the chain base described in 4.9, there is also the chain base $\left(e_{0}, f_{0}\right), \cdots,\left(e_{0}, f_{m-n}\right),\left(e_{1}, f_{m-n+1}\right),\left(e_{2}, f_{m-n+2}\right)$, $\cdots,\left(e_{n-1}, f_{m-1}\right)$. These remarks lead to the next theorem. 
TheORem 4.12. A distributive lattice $A$ is a Post algebra of order $n$ if and only if $A$ has a unique n-term chain base.

Proof. Let $A$ be a Post algebra of order $n$, and let $e_{0}, \cdots, e_{n-1}$ be an $n$-term chain base. $A$ is a subdirect power of an $n$ element chain $C$. If $f_{j}=A \rightarrow C$ is the $j$ th projection, then by $4.10, f_{j}\left(e_{0}\right)$, $\cdots, f_{j}\left(e_{n-1}\right)$ is a chain base of $C$. This determines $f_{j}\left(e_{i}\right)$ uniquely for all $i, j$. Therefore $e_{0}, \cdots, e_{n-1}$ is unique.

Conversely, suppose $A$ has a unique $n$-term chain base $e_{0}, \cdots, e_{n-1}$. We prove $A$ is a Post algebra of order $n$ by induction. This is obvious for $n=1,2$. Suppose $n>2$ and the statement holds for $n-1$. By 2.5, $\left[e_{1}, 1\right]$ has a unique chain base with $n-1$ terms. Therefore, $\left[e_{1}, 1\right]$ is a Post algebra of order $n-1$. This implies $e_{i+1} \Rightarrow$ $e_{\imath}=0$ in $\left[e_{1}, 1\right]$ for $i \geqq 1$. This implies $e_{i+1} \Rightarrow e_{i}=0$ in $A$ since the center of $\left[e_{1}, 1\right]$ is $\left\{b \vee e_{1}: b \in B\right\}$, where $B$ is the center of $A$. By 4.8, we need only show $e_{1} \Rightarrow 0=0$. If not, there exists $b \in B$ with $b e_{1}=0$, $b \neq 0$. Let $B_{1}=\{0, b, \bar{b}, 1\}$, and let $A_{1}$ be the sublattice of $A$ generated by $B_{1} \cup\left\{e_{0}, \cdots, e_{n-1}\right\}$. By $2.2, A_{1}$ has center $B_{1}$ and so every chain base of $A_{1}$ is a chain base of $A$. Thus $A_{1}$ is a finite lattice with a unique $n$-term chain base. By $4.11, A_{1}$ is a direct product of finite chains. If all the chains have the same cardinal, then $A_{1}$ is a Post algebra with unique $n$-term chain base $e_{0}, \cdots, e_{n-1}$, and by 4.8 , we have $e_{1} \Rightarrow 0=0$, which contradicts $b e_{1}=0, b \neq 0$. If two of the chains have different cardinal, then $A_{1}$ has more than one $n$-term chain base. This contradiction proves $e_{1} \Rightarrow 0=0$.

TheOREM 4.13. If $\left\langle A ; e_{0}, \cdots, e_{n-1}\right\rangle$ is a $P_{1}$-lattice with center $B$, then there exists a $P_{2}$-lattice $\left\langle A^{\prime} ; e_{0}, \cdots, e_{n-1}\right\rangle$ with center $B^{\prime}$ such that $B$ is a Boolean subalgebra of $B^{\prime}$ and $A$ is the sublattice of $A^{\prime}$ generated by $B \cup\left\{e_{0}, \cdots, e_{n-1}\right\}$.

Proof. By 3.1, $A$ is an $L$-algebra. By [9], we may assume $A$ is a Heyting subalgebra of a direct product of chains $C_{j}, j \in J$ and the projections $f_{j}: A \rightarrow C_{j}$ are onto. Then by $4.10,\left\langle C_{j} ; f_{j}\left(e_{0}\right), \cdots, f_{j}\left(e_{n-1}\right)\right\rangle$ is a $P_{1}$-lattice. Therefore, $C_{j}$ has at most $n$ elements and $\left\langle C_{j} ; f_{j}\left(e_{0}\right)\right.$, $\left.\cdots, f_{j}\left(e_{n-1}\right)\right\rangle$ is a $P_{2}$-lattice. Let $A^{\prime}=\prod_{j \in J} C_{j}$. By $4.9,\left\langle A ; e_{0}, \cdots, e_{n-1}\right\rangle$ is a $P_{2}$-lattice. Since $A$ is a sublattice of $A^{\prime}$ containing 0,1 , the center of $A$ is a subalgebra of the center of $A^{\prime}$.

THEOREM 4.14. Let $\left\langle A ; e_{0}, \cdots, e_{n-1}\right\rangle$ be a $P_{2}$-lattice of order $n$ with center $B$. Then $A$ is order isomorphic with a direct product of Post algebras of maximum order $n$.

Proof. Let $u_{k}=! e_{k}-! e_{k-1}, 1 \leqq k \leqq n-1$. Then $u_{j} u_{k}=0$ for 
$j \neq k$, and $u_{1} \vee \cdots \vee u_{n-1}=1$. Let $P_{k}=\left[0, u_{k}\right]$. Clearly the center of $P_{k}$ is $B \cap P_{k}$. Let $e_{k i}=e_{i} u_{k}, 0 \leqq i \leqq k$. If $x=\bigvee_{i=1}^{n-1} b_{i} e_{i}$ is a mon. rep. of any $x \in P_{k}$, then

$$
x-x u_{k}=\bigvee_{i=1}^{n-1} b_{i} e_{i} u_{k}=\bigvee_{i=1}^{k-1} b_{i} e_{i} u_{k} \bigvee \bigvee_{i=k}^{n-1} b_{i} u_{k}=\bigvee_{i=1}^{k}\left(b_{i} u_{k}\right) e_{k i} .
$$

Therefore $\left\langle P_{k} ; e_{k 0}, \cdots, e_{k k}\right\rangle$ is a $P_{0}$-lattice. If $b \in P_{k} \cap B, b e_{k i} \leqq e_{k(i-1)}$, $0<i \leqq k$, then $b e_{i} \leqq e_{i-1}$. Therefore $b \leqq e_{i} \Rightarrow e_{i-1}=! e_{i-1}$. This implies $b=0$, since $b \leqq u_{k}$. Thus by $4.8, P_{k}$ is a Post algebra of order $k+1$, or else $P_{k}=\{0\}$. Define $f: A \rightarrow \prod_{k=1}^{n-1} P_{k}$ by $f(x)=\left(x u_{1}\right.$, $\left.\cdots, x u_{n-1}\right) . \quad f$ is onto since if $z_{i} \in P_{i}$, then $f\left(z_{1} \vee \cdots \vee z_{n-1}\right)=\left(z_{1}, \cdots, z_{n-1}\right)$. If $x \leqq y$ then $f(x) \leqq f(y)$, and $f(x) \leqq f(y)$ implies

$$
x=\bigvee_{i=1}^{n-1} x u_{i} \leqq \bigvee_{i=1}^{n-1} y u_{i}=y \text {. }
$$

Therefore $f$ is an order isomorphism. Finally, $P_{n-1}$ has order $n$ since $u_{n-1} \neq 0$.

Theorem 4.14 may be used to apply known results on Post algebras to $P_{2}$-lattices. For example, since every Post algebra is isomorphic with the set of all continuous functions on a Boolean space to a finite discrete chain, it follows that every $P_{2}$-lattice is isomorphic with the set of all such functions which are $\leqq$ some fixed continuous function. In other words, a $P_{2}$-lattice is a principal ideal in a Post algebra. It also follows from 4.14 that a $P_{2}$-lattice is complete if and only if its center is complete, and that the normal completion of a $P_{2}$-lattice $A$ is a $P_{2}$-lattice whose center is the normal completion of the center of $A$. Also every $P_{2}$-algebra is isomorphic with its dual. This isomorphism is given explicitly in the following theorem.

THEOREM 4.15. Let $\left\langle A ; e_{0}, \cdots, e_{n-1}\right\rangle$ be a $P_{2}$-lattice. Let $f_{i}=$ $\mathbf{V}_{k=1}^{n-1-i} e_{k} \overline{e_{k+i-1}}, 0 \leqq i<n-1$ and $f_{n-1}=0$. Then $A$ is isomorphic with $A^{d}$ under the normal involution

$$
\left.\beta(x)=\bigvee_{j=1}^{n-1} \overline{D_{j}(x)} f_{j-1}=\bigwedge_{j=1}^{n-1} \overline{\left(D_{j}(x)\right.} \vee f_{j}\right) .
$$

Proof. We have $f_{0} \geqq \mathbf{V}_{k=1}^{n-1}\left(e_{k}-! e_{k-1}\right)=1$. For $0<i<n-1$, $! f_{i}=0$, so that by $4.5(\mathrm{i}), D_{k}\left(f_{i}\right)=\overline{! e_{k+i-1}}$ for $1 \leqq k \leqq n-1-i$, and $D_{k}\left(f_{i}\right)=0$ for $k \geqq n-i$.

If $1 \leqq i \leqq n-2$,

$$
\begin{aligned}
\beta\left(f_{i}\right) & =\bigvee_{j=1}^{n-1} f_{j-1} \overline{D_{j}\left(f_{i}\right)}=\bigvee_{j=1}^{n-1-i} f_{j-1} ! e_{j+i-1} \bigvee \bigvee_{j=n-i}^{n-1} f_{j-1} \\
& =\bigvee_{j=1}^{n-1} f_{j-1} ! e_{j+\imath-1}=\bigvee_{j=1}^{n-i} ! e_{j+i-1} \bigvee_{k=1}^{n-j} e_{k} \overline{! e_{j+k-2}} \\
& =\bigvee_{k=1}^{i} e_{k} \bigvee_{j=1}^{n-i}\left(! e_{j+i-1}-! e_{j+k-2}\right) \bigvee \bigvee_{k=i+1}^{n-1} e_{k} \bigvee_{j=1}^{n-k}\left(! e_{j+i-1}-! e_{j+k-2}\right) .
\end{aligned}
$$


But $\mathbf{V}_{j=1}^{n-k}\left(! e_{j+i-1}-! e_{j+k-2}\right)=0$ if $k>i$, and by 2.7 , if $k \leqq i$,

$$
\bigvee_{j=1}^{n-i}\left(! e_{j+i-1}-! e_{j+k-2}\right)=\overline{! e_{k-1}} \bigwedge_{j=2}^{n-i}\left(! e_{j+2-2} \vee \overline{! e_{j+k-2}}\right)=\overline{! e_{k-1}}
$$

Therefore, $\beta\left(f_{i}\right)=\mathbf{V}_{k=1}^{i}\left(e_{k}-! e_{k-1}\right)=e_{i}$.

Now $x \leqq y$ implies $\beta(x) \leqq \beta(y)$ and

$$
\beta(\beta(x))=\bigvee_{j=1}^{n-1} f_{j-1} \bigvee_{i=1}^{n-1} D_{i}(x) \overline{D_{j}\left(f_{i}\right)}=\bigvee_{i=1}^{n-1} \beta\left(f_{i}\right) D_{i}(x)=\bigvee_{i=1}^{n=1} D_{i}(x) e_{i}=x
$$

This implies that $\beta: A \rightarrow A^{d}$ is an isomorphism. The proof that $\beta$ is a normal involution - that is, that $x \beta(x) \leqq y \vee \beta(y)$ for all $x, y \in A$ is omitted since this fact will not be used here [10].

5. Axioms and $P_{2}$-functions. $P_{2}$-algebras $\left\langle A ; e_{0}, \cdots, e_{n-1}\right\rangle$ of order $\leqq n$ may be regarded as algebras $\left\langle A ; \vee, \wedge, C_{0}, \cdots, C_{n-1}, e_{0}\right.$, $\left.\cdots, e_{n-1}\right\rangle$ with two binary operations, $n$ binary operations, and $n$ distinguished constants. This class of algebras can be characterized by the following set of equational axioms, in which $x \leqq y$ is used as an abbreviation for $x \wedge y=x$.

H1. Identities characterizing $\langle A ; \vee, \wedge\rangle$ as a distributive lattice $[8$, pp. 5,35$]$.

H2. (a) $e_{0} \leqq x$

(b) $e_{i} \leqq e_{j}$ for $0 \leqq i \leqq j \leqq n-1$

(c) $x \leqq e_{n-1}$

H3. (a) $C_{i}(x) \wedge C_{j}(x)=e_{0}$ for $i \neq j$

(b) $C_{0}(x) \vee C_{1}(x) \vee \cdots \vee C_{n-1}(x)=e_{n-1}$

H4. (a) $C_{i}(x \wedge y)=\left(C_{2}(x) \wedge \bigvee_{\substack{n-1 \\ k=i}} C_{k}(y)\right) \vee\left(C_{i}(y) \wedge \bigvee_{k=i}^{n-1} C_{k}(x)\right)$

(b) $C_{n-1}(x \vee y)=C_{n-1}(x) \vee C_{n-1}(y)$

H5. (a) $C_{i}\left(e_{j}\right)=e_{0}$ for $j \neq i$ and $i<n-1$

(b) $C_{n-1}\left(e_{0}\right)=e_{0}$

H6. $x=\left(C_{1}(x) \wedge e_{1}\right) \vee \cdots \vee\left(C_{n-1}(x) \wedge e_{n-1}\right)$.

Note that in every $P_{2}$-lattice $\mathrm{H} 4$ holds by 4.5 (iv) and $\mathrm{H} 5$ holds by 4.5(ii). Conversely, if $A$ satisfies the axioms then one proves $C_{n-1}(1)=1, C_{0}(0)=0, C_{0}(x)=-x$ and $C_{n-1}(x)=! x$. Then using $\mathrm{H} 4$ and $\mathrm{H} 5$, it can be proved that $x e_{i}=e_{i-1}$ implies $x=e_{i-1}$. This shows that $\left\langle A ; e_{0}, \cdots, e_{n-1}\right\rangle$ is a $P_{2}$-lattice.

The class of Post algebras of order $n$ (together with the trivial lattice $\{0\})$ can be characterized by adding the axiom $C_{n-1}\left(e_{n-2}\right)=0$ (see also [6]).

We may also characterize $P_{2}$-lattices equationally as the class of all algebras $\left\langle A ; \vee, \wedge, \Rightarrow, e_{0}, \cdots, e_{n-1}\right\rangle$ with 3 binary operations and $n$ constants which satisfy the following axioms. 
K1. Identities characterizing $\left\langle A ; \vee, \wedge, \Rightarrow, e_{0}, e_{n-1}\right\rangle$ as a $P$-algebra (see [7]).

K2. $e_{i} \leqq e_{j}$ for $i \leqq j$

K3. $e_{i+1}\left(e_{i} \Rightarrow e_{j}\right) \leqq e_{j}$ for $j<i<n-1$

K4. $\quad x=\bigvee_{j=1}^{n-1}\left(e_{i} \wedge\left(e_{i} \Rightarrow x\right)\right)$.

Indeed, if we set $D_{i}(x)=e_{i} \Rightarrow x$ for $0 \leqq i \leqq n-1$ and let $C_{i}(x)=$ $D_{i}(x)-D_{i+1}(x)$ for $i<n$, then $\mathrm{H} 1-3, \mathrm{H} 5(\mathrm{~b})$, and $\mathrm{H} 6$ are obvious. By properties of $P$-algebras, $D_{i}(x \vee y)=D_{i}(x) \vee D_{i}(y)$ and $D_{i}(x \wedge y)=$ $D_{i}(x) \wedge D_{\imath}(y)$. This proves H4. H5(a) is equivalent to $e_{i} \Rightarrow e_{j}=e_{i+1} \Rightarrow e_{j}$ for $j \neq i, i<n-1$. This is obvious for $i<j$, and follows from $\mathrm{K} 3$ for $i>j$.

$P_{2}$-lattices may also be characterized equationally as algebras $\left\langle A ; \vee, \wedge, \rightarrow, !, e_{0}, \cdots, e_{n-1}\right\rangle$, since $x \Rightarrow y=!(x \rightarrow y), x \rightarrow y=y \vee(x \Rightarrow y)$ and $! x=1 \Longrightarrow x$.

A $P_{2}$-function of order $n$ in $m$ variables is a function built from the identity functions $I_{j}\left(x_{1}, \cdots, x_{m}\right)=x_{j}, 1 \leqq j \leqq m$, and the operations in any of the fundamental sets of operations described above. A normal form for such functions is given in the next theorem.

THeORem 5.1. If $h$ is a $P_{2}$-function of order $n$ in $m$ variables, then

$$
h\left(x_{1}, \cdots, x_{m}\right)=\bigvee_{0 \leqq i_{k} \leqq n-1} h\left(e_{i_{1}}, \cdots, e_{i_{m}}\right) C_{i_{1}}\left(x_{1}\right) \cdots C_{i_{m}}\left(x_{m}\right) .
$$

Proof. The $n^{m}$ terms $C_{i_{1}}\left(x_{1}\right) \cdots C_{i_{m}}\left(x_{m}\right)$ are pairwise disjoint and have join 1 , by axiom H3. By H6, the statement holds when $h$ is one of the identity functions. If the statement holds for $h_{1}$ and $h_{2}$, then it holds for $h_{1} \vee h_{2}$ and $h_{1} \wedge h_{2}$. If it holds for $h$, then it holds for $D_{\imath}(h)$ by 4.5(iv). From this it follows that the statement holds for $C_{j}(h)$.

The normal form in 5.1 was proved for Post algebras in [5], and gives a truth table approach to Post functions. However, in a $P_{2}$-lattice, $h\left(e_{i_{1}}, \cdots, e_{i_{m}}\right)$ is not necessarily in $\left\{e_{0}, \cdots, e_{n-1}\right\}$, as is the case for Post algebras.

6. Applications. $P_{2}$-lattices are of interest in computer science. They can be applied to the theory of machines with $m_{i}$-stable devices, $2 \leqq m_{i} \leqq n$, and to the analysis of machines with 2 -stable devices $Q_{i}$ (flip-flops) whose outputs are discretized as signals in transition $0=e_{0}<e_{1}<\cdots<e_{n-1}=1$. The case $n=3$ is of special interest and is studied in [2] and [3]. $P_{2}$-lattices provide the complete multiple-valued logics for these applications.

$P_{2}$-lattices which admit operations of ring addition and multiplication are of interest in information processing. It is known that 
if $R$ is a ring with unit element which satisfies the identities $x^{p}=x$ and $p x=0$, where $p$ is a prime (so-called $p$-rings [12]), then lattice operations can be defined as polynomials in such a way that $R$ becomes a Post algebra of order $p$. Conversely in any Post algebra of order $p$, ring operations can be defined in terms of the Post operations so that we obtain a $p$-ring. Therefore, direct products of finitely many $p$-rings are $P_{2}$-lattices. Such direct products can be characterized equationally. Indeed one can show that a ring $R$ with unit element is a direct product of rings $R_{1}, \cdots, R_{t}$, where $R_{i}$ is a $p_{i}$-ring and $p_{i} \neq p_{j}$ for $i \neq j$, if and only if $R$ satisfies the following set of identities:

(1) $x^{m}=x$, where $m=1+$ l.c.m. $\left(p_{1}-1, \cdots, p_{t}-1\right)$.

(2) $p_{1} \cdots p_{t} x=0$.

(3) $\left(\prod_{j \neq i} p_{j}\right)\left(x^{p_{i}}-x\right)=0,1 \leqq i \leqq t$.

\section{Prime ideals.}

Definition 7.1. Let $\mathscr{P}(A)$ be the set of prime ideals of $A$. Let $\left\langle A ; e_{0}, \cdots, e_{n-1}\right\rangle$ be a $P_{0}$-lattice with center $B$. If $Q \in \mathscr{P}(B)$ and $1 \leqq k \leqq n-1$, let $P_{k}(Q)=\left\{x \in A: x\right.$ has a mon. rep. $\bigvee_{i} b_{i} e_{i}$ such that $\left.b_{k} \in Q\right\}$. It was proved in [13, Th. 1.5] that either $P_{k}(Q) \in \mathscr{P}(A)$ or $P_{k}(Q)=A$ (the latter possibility was not mentioned). If $P_{k}(Q) \neq A$, then $P_{k}(Q) \cap B=Q$ since if $b \in Q$ then $b=\mathrm{V}_{i} b e_{i} \in P_{k}(Q)$ and prime ideals in $B$ are maximal ideals. If $P \in \mathscr{P}(A)$, then $P$ is said to be of type $k$ if $k$ is the smallest integer such that $e_{k} \notin P$. Since $e_{k-1}=$ $e_{1} \vee \cdots \vee e_{k-1} \in P_{k}(Q), P_{k}(Q)$ is of type $\geqq k$.

Lemma 7.2. If $P$ is a prime ideal of type $k$ in $A$ and $Q=P \cap B$, then

$$
P=P_{k}(Q)=\left\{x: \text { for every mon. rep. } \bigvee_{\imath} b_{i} e_{i} \text { of } x, b_{k} \in Q\right\}
$$

Proof. If $x \in A$ has a mon. rep. $\bigvee_{i} b_{2} e_{i}$ with $b_{k} \in Q$ then $x \leqq$ $e_{k-1} \vee b_{k} \in P$. If $x \in P$ and $\bigvee_{i} b_{\imath} e_{i}$ is any mon. rep. of $x$, then $b_{k} e_{k} \in P$ and $e_{k} \notin P$, so that $b_{k} \in Q$.

Theorem 7.3. The prime ideals of a $P_{0}$-lattice $\left\langle A ; e_{0}, \cdots, e_{n-1}\right\rangle$ lie in disjoint maximal chains with at most $n-1$ members.

Proof. By 7.1, each prime ideal of $A$ is of the form $P_{k}(Q)$. If $P_{k}\left(Q_{1}\right) \subseteq P_{j}\left(Q_{2}\right)$, then $Q_{1}=P_{k}\left(Q_{1}\right) \cap B \subseteq P_{j}\left(Q_{2}\right) \cap B=Q_{2}$, so that $Q_{1}=Q_{2}$. It is obvious that $P_{k}(Q) \subseteq P_{k+1}(Q)$. This proves the theorem.

Lemma 7.4. If $\left\langle A ; e_{0}, \cdots, e_{n-1}\right\rangle$ is a $P_{0}$-lattice with center $B$, and $Q \in \mathscr{P}(B)$, then $P_{k}(Q)=\left\{x:\right.$ for some $\left.b \in Q, x \leqq e_{k-1} \vee b\right\}$. Also 
$P_{k+1}(Q)=P_{k}(Q)$ if and only if $e_{k} \in P_{k}(Q)$.

Proof. If $x \in P_{k}(Q)$, there exists a mon. rep. $\bigvee_{i} b_{i} e_{i}$ of $x$ such that $b_{k} \in Q$. Also $x \leqq e_{k-1} \vee b_{k}$. If $x \leqq e_{k-1} \vee b$ and $b \in Q$ then $x \in P_{k}(Q)$ since $e_{k-1} \bigvee b=\bigvee_{j=1}^{k-1} e_{j} \vee \bigvee_{j=k}^{n-1} b e_{j}$. Suppose $e_{k} \in P_{k}(Q)$. If $x \in P_{k+1}(Q)$, then $x \leqq e_{k} \vee b$ for some $b \in Q$, hence $x \in P_{k}(Q)$. Thus $P_{k+1}(Q)=P_{k}(Q)$. Conversely if $P_{k+1}(Q)=P_{k}(Q)$, then $e_{k} \in P_{k}(Q)$ since $e_{k} \in P_{k+1}(Q)$.

THEOREM 7.5. Let $\left\langle A ; e_{0}, \cdots, e_{n-1}\right\rangle$ be a $P_{0}$-lattice with center $B$, and let $I_{k}$ be the ideal $\left\{b \in B: b e_{k} \leqq e_{k-1}\right\}$ in $B, 1 \leqq k \leqq n-1$. Then the following are equivalent:

(i) Every chain in $\mathscr{P}(A)$ has fewer than $n-1$ elements.

(ii) For every $Q \in \mathscr{P}(B)$, there exists $b \in Q$ and an integer $k \geqq 1$ such that $e_{k} \leqq e_{k-1} \vee b$.

(iii) $I_{1} \vee \cdots \vee I_{n-1}=B$.

(iv) $A$ has a chain base with fewer than $n$ elements.

Proof. (i) implies (ii): If $Q \in \mathscr{P}(B)$, then either $P_{n-1}(Q)=A$ or $P_{k}(Q)=P_{k+1}(Q)$ for some $k<n-1$. Hence by 7.4, $e_{k} \in P_{k}(Q)$ for some $k, 1 \leqq k \leqq n-1$, and therefore there exists $b \in Q$ such that $e_{k} \leqq e_{k-1} \vee b$.

(ii) implies (iii): If $I_{1} \vee \cdots \vee I_{n-1} \neq B$, there exists $Q \in \mathscr{P}(B)$ such that $Q \supseteqq I_{1} \vee \cdots \vee I_{n-1}$. There exists $b \in Q$ and $k$ such that $e_{k} \leqq e_{k-1} \vee b$. But then $\bar{b} \in I_{k} \leqq Q$, which is impossible.

(iii) implies (iv): There exist elements $b_{k} \in I_{k}$ such that $1=b_{1} \vee$ $\cdots \vee b_{n-1}$. By replacing the $b_{k}$ by smaller elements, we may assume the $b_{k}$ are pairwise disjoint. Let $f_{0}=0$ and

$$
f_{k}=e_{k} \vee e_{k+1} \bigvee_{j=1}^{k} b_{j}, \quad 1 \leqq k \leqq n-2 .
$$

Then $f_{n} \leqq f_{n+1}$ and $f_{n-2}=1$, since $b_{1} \vee \cdots \vee b_{n-2}=\bar{b}_{n-1}$ and $b_{n-1} \leqq e_{n-2}$. Now $f_{k-1} \vee f_{k} \bigvee_{j=k+1}^{n-1} b_{j}=e_{k-1} \vee \bigvee_{j \neq k} b_{j}$. Therefore,

$$
e_{k}=f_{k-1} \vee f_{k} \bigvee_{j=k+1}^{n-1} b_{j},
$$

and so $f_{0}, \cdots, f_{n-2}$ is a chain base of $A$.

(iv) implies (i) by 7.3 .

TheOREm 7.6. Let $A$ be a $P_{0}$-lattice. Then $A$ is of order $n$ if and only if the maximum number of elements in a chain in $\mathscr{P}(A)$ in $n-1$.

Proof. This follows from 7.3 and the equivalence of 7.5(i) and 7.5(iv). 
Definition 7.7. Let $\mathscr{P}_{0}(A)=\phi$, and let $\mathscr{P}_{k+1}(A)$ be the set of minimal elements of $\mathscr{P}(A)-\mathscr{\mathscr { P }}_{k}(A)$.

TheOREM 7.8. Let $\left\langle A ; e_{0}, \cdots, e_{n-1}\right\rangle$ be a $P_{1}$-lattice with center $B$. Then for $0 \leqq i \leqq n-2$,

$$
e_{i} \in \bigcap \mathscr{P}_{i+1}(A)-\bigcup_{j \leq i} \bigcup \mathscr{P}_{j}(A) \text {. }
$$

Proof. By 7.4, $P_{1}(Q) \neq A$ for all $Q \in \mathscr{P}(B)$. Hence $\mathscr{P}_{1}(A)=$ $\left\{P_{1}(Q): Q \in \mathscr{P}(B)\right\}$. If $1 \leqq i \leqq n-2$, then $e_{i} \in P_{i}(Q)$ if and only if $e_{i} \leqq e_{i-1} \vee b$ for some $b \in Q$. This in turn is equivalent to $\bar{b} \leqq e_{i} \rightarrow e_{i-1}$ which is equivalent to $\bar{b} \leqq e_{i-1}$, or $1 \leqq e_{i-1} \vee b$. By 7.4, this is equivalent to $P_{i}(Q)=A$. Also, $P_{i}(Q)=P_{i+1}(Q)$ if and only if $e_{i} \in P_{i}(Q)$. Therefore $\mathscr{P}_{i}(A)=\left\{P_{i}(Q): P_{i}(Q) \neq A\right\}$, and $e_{i} \notin P_{i}(Q)$ for all $P_{i}(Q) \in$ $\mathscr{P}_{i}(A)$. Since $e_{i} \in P_{i+1}(Q)$ for all $Q \in \mathscr{P}(B)$, the proof is complete.

Lemma 7.9. Let $A$ be a bounded distributive lattice. Suppose $\mathscr{P}(A)$ is a union of disjoint maximal chains and there exists an element $e \in \bigcap\left(\mathscr{P}(A)-\mathscr{P}_{1}(A)\right)-\mathrm{U} \mathscr{P}_{1}(A) . \quad$ Let $A_{1}=[e, 1] . \quad$ Then $\mathscr{P}_{i}\left(A_{1}\right)=\left\{P \cap A_{1}: P \in \mathscr{P}_{i+1}(A)\right\}$ for each $i \geqq 1$.

Proof. If $P \in \mathscr{P}(A)-\mathscr{P}_{1}(A)$, let $\varphi(P)=P \cap A_{1}$. Then $\varphi(P) \in$ $\mathscr{P}\left(A_{1}\right)$. If $Q \in \mathscr{P}\left(A_{1}\right)$, let $\psi(Q)=\{x \in A: x \geqq$ an element of $Q\}$. Then $\psi(Q) \in \mathscr{P}(A)-\mathscr{P}_{1}(A)$ and $\psi \varphi(P)=P$. Thus $\varphi: \mathscr{P}(A)-\mathscr{P}_{1}(A) \rightarrow \mathscr{P}\left(A_{1}\right)$ is an order isomorphism.

Lemma 7.10. Under the hypotheses of 7.9, let $B$ and $B_{1}$ be the centers of $A$ and $A_{1}$ respectively. Then $B_{1}=\{b \vee e: b \in B\}$. If $x \in A$, then there exists $b \in B$ such that $x=b(e \vee x)$.

Proof. Let $\left\{D_{i}: i \in S\right\}$ be the set of maximal chains in $\mathscr{P}(A)$. The intersection and union of any nonempty subset of $D_{i}$ is in $D_{i}$. Let $P_{i}$ and $Q_{i}$ be respectively the smallest and largest member of $D_{i}$. Let $V=\left\{i: P_{i} \neq Q_{i}\right\}$. For $i \in V$, let $R_{i}=\bigcap\left\{P \in D_{i}: e \in P\right\} . \quad R_{i}$ is the immediate successor of $P_{i}$ in $D_{i}$. We divide the proof of the lemma into several parts.

(a) If $x \in P_{i}$, there exists $y$ such that $x y=0$ and $y \notin Q_{i}$.

Indeed for each $j$ such that $x \notin P_{j}$, choose $y_{j} \in P_{j}-Q_{i}$. Then every prime ideal in $A$ contains a member of $\{x\} \cup\left\{y_{j}: x \notin P_{j}\right\}$. Therefore, the filter generated by this set is not proper and so there exists a finite meet $y$ of the $y_{j}$ such that $x y=0$. Clearly $y \notin Q_{i}$.

(b) If $x \notin Q_{i}$, there exists $y \in P_{i}$ such that $x \vee y=1$.

For each $j$ such that $x \in Q_{j}$ choose $y_{j} \in P_{i}-Q_{j}$. The ideal generated by $\{x\} \vee\left\{y_{j}: x \in Q_{j}\right\}$ is not proper. Therefore, a finite join 
$y$ of the $y_{j}$ satisfies the requirements.

(c) If $x \notin Q_{i}$ there exists $y \leqq x$ such that $y \notin Q_{i}$ and $y \in P_{j}$ whenever $x \in Q_{j}$.

By (b) there exists $z \in P_{i}$ such that $x \vee z=1$. By (a) there exists $y \notin Q_{i}$ such that $y z=0$. If $x \in Q_{j}$, then $z \notin Q_{j}$, hence $z \notin P_{j}$ and so $y \in P_{j}$. If $P$ is any prime ideal containing $x$ then $P \in D_{j}$ for some $j$, and so $x \in Q_{j}$. This implies $y \in P_{j} \subseteq P$. Thus $y \leqq x$.

(d) If $x \notin P_{i}$, there exists $y \notin Q_{i}$ such that $e y \leqq x$.

For each $j$ such that $x \in P_{j}$, choose $y_{j} \in P_{j}-Q_{i}$. If $P$ is a prime ideal containing $x$ but not $e$, then $P=P_{j}$ for some $j$ and so $y_{j} \in P$. This implies that $x$ belongs to the filter generated by $\{e\} \vee\left\{y_{j}: x \in P_{j}\right\}$. The desired $y$ will be the meet of a finite number of $y_{j}$.

(e) If $x \in R_{i}$, there exists $y \notin Q_{i}$ such that $x y \leqq e$.

For each $j$ such that $x \notin R_{j}$ choose $y_{j} \in P_{j}-Q_{i}$. If $P$ is a prime ideal containing $e$ but not $x$, then $P \supseteqq R_{j}$ for some $j$ and since $x \notin R_{j}$, $y_{j} \in P_{j} \subseteq P$. This implies that $e$ belongs to the filter generated by $\{x\} \vee\left\{y_{j}: x \notin R_{j}\right\}$. The desired $y$ is the meet of a finite number of $y_{j}$.

(f) If $x \in B_{1}$ then for all $i$, either $x \in R_{i}$ or $x \notin Q_{i}$.

Let $y$ be the complement of $x$ in $A_{1}$. If $x \in Q_{i}$ then $y \notin Q_{i}$ since $x \vee y=1$. Therefore $y \notin R_{i}$, hence $x_{i} \in R_{i}$ since $x y=e \in R_{i}$.

(g) If for all $i, x \in P_{i}$ or $x \notin Q_{i}$, then $x \in B$.

By (a), for each $i$ such that $x \in P_{i}$, there exists $y_{i} \notin Q_{i}$ such that $x y_{i}=0$. No prime ideal contains $x$ and $\left\{y_{i}: x \in P_{\imath}\right\}$. There exists a finite join $y$ of the $y_{i}$ such that $x \vee y=1$ and clearly $x y=0$.

(h) If $x \in A$, there exists $y \in B$ such that $x=y(e \vee x)$.

Let $T=\left\{j: x \in P_{j}\right\}$. If $T=S$ then $x=0$ and $y=0$. If $T=\phi$ then $x \geqq e$ and $y=1$ will do. Suppose $T \neq S, T \neq \phi$. By (d), for each $i \in S-T$, there exists $y_{i} \notin Q_{i}$ such that $e y_{i} \leqq x$. By (a), for each $j \in T$ there exists $z_{j} \notin Q_{j}$ such that $x z_{j}=0$. No prime ideal contains $\left\{y_{i}: i \in S-T\right\} \cup\left\{z_{j}: j \in T\right\}$. Therefore, there exist $y, z$ such that $y \vee z=1, e y \leqq x$, and $x z=0$. This implies $x=x y=x y \vee e y=$ $x(y \vee e)$. If $j \in T$, then $e y \leqq x \in P_{j}, e \notin P_{j}$ so that $y \in P_{j}$. If $i \in S-T$. then $z \in P_{i}$ since $x \notin P_{i}$ and $x z=0$. Thus $y z \in P_{i}$ for all $i \in S$, and so $y z=0$. Hence $y \in B$.

(i) If $x \in B_{1}$, there exists $y \in B$ such that $x=y \vee e$.

Let $W=\left\{j \in V: x \in R_{j}\right\}$. If $W=V$, then $x=e$ and $y=0$. If $W=\phi$ then by (f), $x=1$ and $y=1$. Suppose $W \neq V, W \neq \phi$. By (c), for each $i \in S-W$ there exists $y_{i} \leqq x$ such that $y_{i} \notin Q_{i}$ and $y_{i} \in P_{j}$ for all $j \in W$. By (e), for each $j \in W$, there exists $z_{j} \notin Q_{j}$ such that $x z_{j} \leqq e$. No prime ideal contains $\left\{y_{i}: i \in S-W\right\} \vee\left\{z_{j}: j \in W\right\}$. Therefore, there exist $y, z$ such that $1=y \vee z, x z \leqq e, y \leqq x$ and $y \in P_{j}$ for all $j \in W$. If $i \in V-W$ then $x \notin R_{i}$ and $e \in R_{i}$, hence $z \in R_{i}$ and so $y \notin R_{i}$. If $i \in S-V$, then $y \in P_{i}$ or $y \notin Q_{i}$ since $P_{i}=Q_{i}$. Therefore by $(\mathrm{g}), y \in B$. Finally $y \vee e \leqq x=x y \vee x z \leqq y \vee e$, and so $x=y \vee e$. 
(h) and (i) yield the lemma since it is obvious that $\{b \vee e: b \in B\} \subseteq B_{1}$.

TheOREm 7.11. Let $A$ be a bounded distributive lattice. Suppose $\mathscr{P}(A)$ is a union of disjoint maximal chains with maximum number of elements equal to $n-1$, and for each $i, 0 \leqq i \leqq n-2$, there exists an element $e_{i} \in \bigcap \mathscr{P}_{i+1}(A)-\bigcup_{j \leqq i} \cup \mathscr{P}_{j}(A)$. If we set $e_{n-1}=1$, then $\left\langle A ; e_{0}, \cdots, e_{n-1}\right\rangle$ is a $P_{1}$-lattice.

Proof. Clearly $0=e_{0}<e_{1}<\cdots<e_{n-2}<1$. If $n=2$, then $A$ is a Boolean algebra by Nachbin's theorem [8, p. 76], and the theorem holds. Assume $n>2$ and the theorem holds for $n-1$. Let $A_{1}=$ $\left[e_{1}, 1\right]$. By 7.9, $A_{1}$ satisfies the hypothesis for $n-1$. Therefore $\left\langle A_{1} ; e_{1}, \cdots, e_{n-1}\right\rangle$ is a $P_{1}$-lattice. Let $x$ be any member of $A$. By 7.10, $x \vee e_{1}=\bigvee_{i=2}^{n-1}\left(e_{1} \vee b_{i}\right) e_{i}$, where $b_{2} \in B$. Again by 7.10, there exists $b \in B$ such that $x=b\left(x \vee e_{1}\right)$. Therefore $x=b e_{1} \vee \bigvee_{i=2}^{n-1} b b_{i} e_{i}$. Clearly $e_{i+1} \rightarrow e_{i}=e_{i}$ in $A$, for $i \geqq 1$. It remains to show $e_{1} \rightarrow 0=0$. Suppose $y e_{1}=0$ and $y \neq 0$. There exists a maximal filter $F$ containing $y$. But $A-F \in \mathscr{P}_{1}(A)$, and so $e_{1} \in F$. Thus $0 \in F$, a contradiction.

Theorem 7.12. Let $A$ be a bounded distributive lattice. Then there exists a sequence $e_{0}, \cdots, e_{n-1}$ such that $\left\langle A ; e_{0}, \cdots, e_{n-1}\right\rangle$ is a $P_{1}$-lattice of order $n$ if and only if

(i) $\mathscr{P}(A)$ is a union of disjoint maximal chains with maximum number of elements equal to $n-1$, and

(ii) $\cap \mathscr{S}_{i+1}(A)-\mathrm{U}_{j \leqq \imath} \mathrm{U} \mathscr{P}_{j}(A) \neq \phi$.

Proof. This follows from 7.6, 7.8, and 7.11.

THEOREM 7.13. Let $A$ be a bounded distributive lattice. Then there exists a sequence $e_{0}, \cdots, e_{n-1}$ such that $\left\langle A ; e_{0}, \cdots, e_{n-1}\right\rangle$ is a $P_{2}$-lattice of order $n$ if and only if conditions (i) and (ii) of Theorem 7.12 hold as well as

(iii) There exists an element $c \in A$ such that for all $P \in \mathscr{P}(A)$, $c \in P$ if and only if $P$ is a maximal ideal.

Proof. By the equivalence of (i) and (iii) in Theorem 4.6, this is a consequence of $[11,4.9]$.

A characterization of Post algebras $A$ by properties of $\mathscr{P}(A)$ is known [4]. However, we know no such characterization of $P_{0}$-lattices. We give an example of a $P$-algebra $A$ such that $\mathscr{P}(A)$ consists of disjoint maximal chains with at most 2 elements but $A$ is not a $P_{0}$-lattice. Let $C=\{0, e, 1\}$ be a 3 element chain and let $A$ be the set of all functions $f$ on an infinite set $I$ to $C$ such that $\{C: f(i)=e\}$ is finite. Since $A$ is a $P$-subalgebra of a Post algebra of order 3, 
each chain of prime ideals of $A$ has length at most 2, [7, Th. 7.1]. If $0=f_{0}<f_{1}<\cdots<f_{n-1}=1$ and $S_{k}=\left\{i: f_{k}(i)=e\right\}$, and if $f=\mathbf{V}_{i=1}^{n-1} b_{i} f_{i}$, where $b_{i}$ are in the center of $A$, then $\{i: f(i)=e\} \leqq S_{1} \cup \cdots \cup S_{n-1}$. Therefore, $A$ does not have a chain base.

\section{REFERENCES}

1. R. Balbes and A. Horn, Stone lattices, Duke Math. J., 38 (1970), 537-545.

2. R. Braddock, G. Epstein, and H. Yamanaka, Multiple-valued logic design and applications in binary computeers, Conference Record of the 1971 Symposium on the Theory and Applications of Multiple-Valued Logic Design, Buffalo, (1971), 13-25.

3. M. Breuer and G. Epstein, The smallest many-valued logic for the treatment of complemented and uncomplemented error signals, Conference Record of the 1973 International Symposium of Multiple-Valued Logic, Toronto, (1973), 29-37.

4. C. Chang and A. Horn, Prime ideal characterization of generalized Post algebras, Proc. of Symposia in Applied Mathematics, Amer. Math. Soc., 2 (1961).

5. G. Epstein, The lattice theory of Post algebras, Trans. Amer. Math. Soc., 95 (1960), 300-317.

6. - An equational axiomatization for the disjoint system of Post algebras, IEEE Transactions on Computers, C-22 (1973), 422-423.

7. G. Epstein and A. Horn, P-algebras, an abstraction from Post algebras, Algebra Universalis, (to appear).

8. G. Grätzer, Lattice Theory, San Francisco, 1971.

9. A. Horn, Logic with truth values in a linearly ordered Heyting algebra, J. Symbolic Logic, 34 (1969), 395-408.

10. J. Kalman, Lattices with involution, Trans, Amer. Math. Soc., 87 (1958), 485-491.

11. T. Katriňák und A. Mitscke, Stonesche verbände der ordnung $n$ und Postalgebren, Mathematische Annalen, 199 (1972), 13-30.

12. N. McCoy and D. Montgomery, A representation of generalized Boolean rings, Duke Math. J., 3 (1937), 455-459.

13. T. Traczyk, Axioms and some properties of Post algebras, Colloquium Mathematicum, 10 (1963), 193-209.

Received March 15, 1974. This work was supported in part by the Office of Naval Research under Contract N00014-67-A-0269-0019, NR 048-299.

UNIVERSiTY OF SOUTHERN CALIFORNIA,

INDIANA UNIVERSITY AND

University of California, Los ANgeles 


\section{PACIFIC JOURNAL OF MATHEMATICS}

EDITORS

RICHARD ARENS (Managing Editor)

University of California

Los Angeles, Calıfornia 90024

R. A. Beaumont

University of Washington

Seattle, Washington 98105
J. DugundJI

Department of Mathematics

University of Southern California

Los Angeles, California 90007

D. Gilbarg and J. Milgram

Stanford University

Stanford, California 94305

\section{ASSOCIATE EDITORS}
E. F. BECKENBACH
B. H. NeumanN
F. WOLF
K. YOSHIDA

\section{SUPPORTING INSTITUTIONS}

UNIVERSITY OF BRITISH COLUMBIA

CALIFORNIA INSTITUTE OF TECHNOLOGY

UNIVERSITY OF CALIFORNIA

MONTANA STATE UNIVERSITY

UNIVERSITY OF NEVADA

NEW MEXICO STATE UNIVERSITY

OREGON STATE UNIVERSITY

UNIVERSITY OF OREGON

OSAKA UNIVERSITY
UNIVERSITY OF SOUTHERN CALIFORNIA

STANFORD UNIVERSITY

UNIVERSITY OF TOKYO

UNIVERSITY OF UTAH

WASHINGTON STATE UNIVERSITY

UNIVERSITY OF WASHINGTON

AMERICAN MATHEMATICAL SOCIETY NAVAL WEAPONS CENTER 


\section{Pacific Journal of Mathematics}

\section{Vol. 55, No. $1 \quad$ September, 1974}

Robert Lee Anderson, Continuous spectra of a singular symmetric

differential operator on a Hilbert space of vector-valued functions . . . $\quad 1$

Michael James Cambern, The isometries of $L^{p}(X, K) \ldots \ldots \ldots \ldots \ldots . . \ldots$

R. H. Cameron and David Arne Storvick, Two related integrals over spaces of continuous functions ................................

Gary Theodore Chartrand and Albert David Polimeni, Ramsey theory and

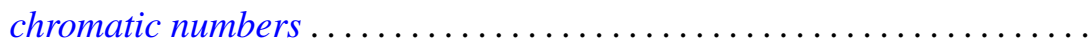

John Deryck De Pree and Harry Scott Klein, Characterization of collectively compact sets of linear operators ...................

John Deryck De Pree and Harry Scott Klein, Semi-groups and collectively compact sets of linear operators ....................... 55

George Epstein and Alfred Horn, Chain based lattices.............. 65

Paul Erdős and Ernst Gabor Straus, On the irrationality of certain series . . 85

Zdeněk Frolík, Measurable uniform spaces................... 93

Stephen Michael Gagola, Jr., Characters fully ramified over a normal

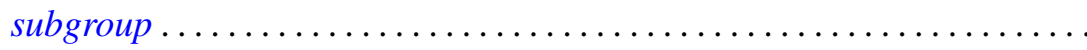

Frank Larkin Gilfeather, Operator valued roots of abelian analytic

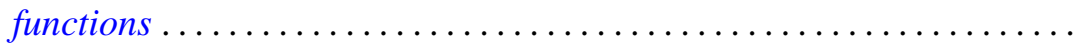

D. S. Goel, A. S. B. Holland, Cyril Nasim and B. N. Sahney, Best approximation by a saturation class of polynomial operators

James Secord Howland, Puiseux series for resonances at an embedded

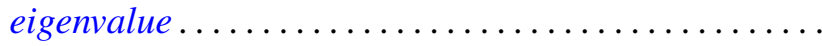

David Jacobson, Linear GCD equations .................

P. H. Karvellas, A note on compact semirings which are multiplicative

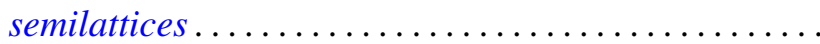

Allan Morton Krall, Stieltjes differential-boundary operators. II . .

D. G. Larman, On the inner aperture and intersections of convex sets

S. N. Mukhopadhyay, On the regularity of the $P^{n}$-integral and its application to summable trigonometric series ....... .

Dwight Webster Read, On $(J, M, m)$-extensions of Boolean algebras ....

David Francis Rearick, Multiplicativity-preserving arithmetic power series.

Indranand Sinha, Characteristic ideals in group algebras

Charles Thomas Tucker, II, Homomorphisms of Riesz spaces . . .

Kunio Yamagata, The exchange property and direct sums of indecomposable injective modules. 